\title{
NEURAL NETWORKS APPLIED TO THE CONTROL OF A FOUR-WIRE SHUNT ACTIVE POWER FILTER
}

\author{
Marcelo G. Villalva, Ernesto Ruppert F. \\ FEEC - UNICAMP \\ CEP 13083 - 852, Caixa Postal 6101, Campinas - SP, Brasil \\ mvillalv@dsce.fee.unicamp.br,ruppert@fee.unicamp.br
}

\begin{abstract}
This paper presents a four-wire shunt active power filter fully controlled with neural networks. The paper is centered on a current compensation method based on adaptive linear elements (adalines), which are powerful and easy-to-use neural networks. The reader will find here an introduction about these networks, an explanatory section about the achievement of Fourier series with adalines, and the full description of an adaline-based selective current compensator. The paper also brings a discussion about the use of a feedforward neural network in the current controller of the active filter, as well as simulation and experimental results obtained with a prototype of a shunt active power filter.
\end{abstract}

Keywords - active filter, adaline, neural network

\section{INTRODUCTION}

Active power filter control strategies have been extensively studied in the past decades. Along the recent years many authors have presented alternative control schemes for the compensation of harmonic and reactive currents with active power filters but very little was said about four-wire applications. Many low voltage and medium power distribution systems feed unbalanced loads with a neutral conductor. An active filter employed in such systems, for filtering both harmonic and reactive currents, needs a fourth wire in order to achieve full compensation of the neutral current.

The possibility of decreasing the size of electronic power converters while keeping the current harmonic distortion within preset limits has increased the interest in selective compensation. A simple selective compensation method for active power filters based on neural networks was studied in [1-3], but the authors have not discussed either the compensation of unbalanced currents in four-wire systems, or the compensation of the fundamental harmonic reactive currents. In addition references [1-3] lack discussions about synchronism and immunity to voltage distortions and frequency deviations, which may cause malfunction of the active power filter system. Other compensation methods have been proposed by several authors. In [4] the authors achieve selective current compensation with discrete Fourier transform-based filters. In [5] another selective compensation method based on synchronous reference frame coordinates is proposed.

Artigo submetido em 3/2/2005. Primeira revisão em 25/3/2005. Aceito sob recomendação dos editores especiais Marcelo G. Simões e Humberto Pinheiro.
This paper studies a current compensator for four-wire shunt active power filters based on adaptive linear elements (adalines), which are simple and very powerful neural networks. This current compensator uses three adalines to achieve selective compensation of harmonic currents in three-phase electric systems with neutral conductor.

Neural networks are proven to be very easy-to-use systems, with innumerable possibilities of application in all majors of engineering and science. Moreover, their design and implementation are frequently very simple and require little programming effort. The objective of this paper is to study and present the application of adaptive neural network technology to a power electronics problem which is generally solved by other traditional means.

In the following sections the reader is introduced to the adaptive linear neural network theory and learns how it can be used in the development of a current compensator for four-wire shunt active power filters, which is the main contribution of this paper. After necessary and enough theoretical issues, simulated and practical results are shown.

\section{ADALINES}

Adalines are adaptive linear elements well known from the theory of neural networks [6-9]. An adaline is a neural network composed of only one neuron, which is the adaline itself. Fig. 1 shows an adaline: it is composed of an input vector, a weight vector and an activation function. The weight vector $\boldsymbol{W}=\left[\begin{array}{llll}w_{1} & w_{2} & \ldots & w_{N}\end{array}\right]^{\mathrm{T}}$ corresponds to the set of synaptic strengths of the neuron. The input vector $\boldsymbol{X}=\left[\begin{array}{ll}x_{1} & x_{2}\end{array}\right.$ ... $\left.x_{N}\right]^{\mathrm{T}}$ corresponds to the set of input stimuli of the neuron. The activation function $y=f(v)$ represents the behavior of the neuron core. Different activation functions are possible for artificial neural networks. The adaline neuron uses the linear activation function $f(v)=v$, so the output $y$ of the neuron is given by the sum of the weighted inputs $y=w_{1} x_{1}$ $+w_{2} x_{2}+\ldots+w_{N} x_{N}$.

When a neuron is excited it produces the output $y$, which depends on its inputs and on the state of the weight vector. If a neuron is intensely or frequently excited it suffers synaptic or weight modifications. The $\boldsymbol{W}$ vector may be constantly modified during the life of the neuron or during the training or learning process. In the theory of artificial neural networks the learning process may be divided into two major categories: online and offline. The adaline is an online trained neuron whose training process is carried at every instant of time. Other kinds of neural networks are trained only once so that the network is effectively used only after a fixed set of synaptic weights is obtained (generally after a long and complex training process). 


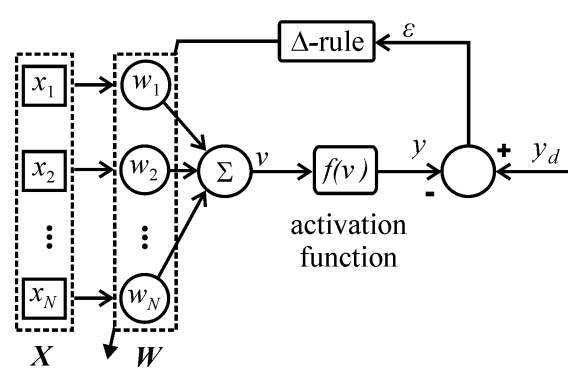

Fig. 1: Adaptive linear element (adaline).

A learning rule must be used to update or to modify the weight vector $\boldsymbol{W}$ of the neuron. Since neurons are generally part of a more complex system that is capable to learn or to acquire knowledge the process of weight updating is referred to as a learning process. One says that a neuron is undergoing a learning process when $\boldsymbol{W}$ is being updated. Adalines are trained by an online learning process based on the $\Delta$-rule, which is an error minimization algorithm. This is a very interesting subject in the field of neural networks that is better studied and explained in works specifically related to the theory of neural networks. An accurate and deep insight into this subject is not in the scope of this text. For the purposes of this paper it is enough to know that the $\Delta$-rule is given by equation (1), where the index $k$ corresponds to the instant $k T$ ( $T$ is the sampling interval of the $\Delta$-rule algorithm), $\alpha$ is the learning rate of the neuron, $\varepsilon$ is the error between the output of the neuron and the desired output $(\varepsilon=$ $\left.y_{d}-y\right)$, and $\boldsymbol{X}(k)$ is the input vector.

$$
\boldsymbol{W}(k+1)=\boldsymbol{W}(k)+\alpha \varepsilon \frac{\boldsymbol{X}(k)}{\boldsymbol{X}^{T}(k) \boldsymbol{X}(k)}
$$

The learning rate $\alpha$ is an important parameter. A high value of $\alpha$ increases the learning speed of the adaline but cannot warranty an accurate learning (i.e. the minimum error between the output and the desired output may be too high). A small value of $\alpha$, on the other hand, decreases the learning speed but can provide a small convergence error. Generally the value of $\alpha$ is chosen on an empiric basis [7]. More elegant methods for the determination of $\alpha$ do exist, but they do not apply to all practical situations. Reference [7] shows how $\alpha$ can be mathematically calculated for an adaline with random inputs, which of course is not the case of the adaline studied in this paper. For most applications $\alpha$ can be empirically determined without loss of scientific rigour.

\section{ADAPTIVE FOURIER SERIES}

In the previous section one learned that the weights of the adaline are updated with the $\Delta$-rule so that the error $\varepsilon$ becomes and remains minimum. As a consequence of a low $\varepsilon$ (when the adaline is said to be converged) the output $y$ of the adaline follows the desired output $y_{d}$. If $y_{d}$ is a time-varying signal the adapting or learning process must be carried in real time and must be fast enough in order to produce a small error at every time instant. If $y_{d}$ is a periodic signal the

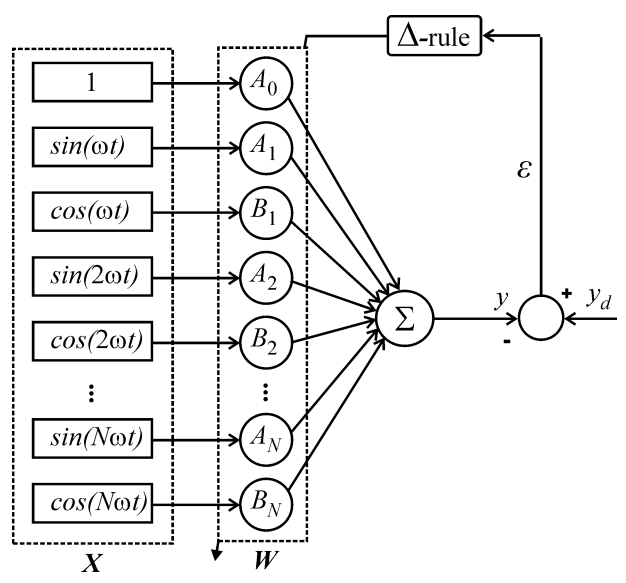

Fig. 2: Fourier series with adaline.

adaline gets (after a brief adapting process) a small error $\varepsilon$ and a quasi-stationary vector $\boldsymbol{W}$. When the error becomes minimum one says that the adaline is trained or adapted. When the waveform of the signal $y_{d}$ is modified the adaline undergoes another training process so that it can learn again how to reproduce $y_{d}$. When the error becomes again minimum the adaline gets adapted to the new condition. Whenever a steady state condition is reached the adaline is adapted and $y \approx y_{d}$.

The adaptive behavior of the adaline can be used to determine the approximate Fourier series of the signal $y_{d}$. This can be achieved if the inputs of the adaline are trigonometric time functions with fundamental and harmonic frequencies. Fig. 2 shows how this is done.

The truncated Fourier series of the time function $y \approx y_{d}$ is given by equation (2). This series contains $N$ oscillatory components and one constant component. The trigonometric functions have angular frequencies $n \omega$, where $n$ is the harmonic order $(n=1$ corresponds to the fundamental frequency).

$$
y_{d}(k T) \approx A_{0}+\sum_{n=1}^{N} C_{n} \sin \left(n \omega k T+\varphi_{n}\right)
$$

Equation (2) may be rewritten as (3), where every oscillatory component is expressed as a sum of two orthogonal trigonometric functions. Because the compensation method is supposed to be implemented in digital processors it is convenient to write (2) and (3) in their discrete forms, where the index $k$ corresponds to the time instant $k T$. The sampling interval $T$ is the same used in the $\Delta$ rule of equation (1).

$$
y_{d}(k T) \approx A_{0}+\sum_{n=1}^{N} A_{n} \sin (n \omega k T)+\sum_{n=1}^{N} B_{n} \cos (n \omega k T)
$$

When the adaline of Fig. 2 is used to determine the Fourier series of $y_{d}$ the weight vector $\boldsymbol{W}$ undergoes an adaptive updating process with the $\Delta$-rule of (1). If the error $\varepsilon$ is sufficiently small the components of the weight vector are approximations of the coefficients of the truncated Fourier series of the time function $y_{d}$. For simplicity the input vector $\boldsymbol{X}$ (in the discrete form) and the weight vector 
$\boldsymbol{W}$ are written in equations (4) and (5). The meaning of the angle $\phi_{v}$ which appears in (4) is explained in next section.

$$
\begin{aligned}
& \boldsymbol{X}=\left[\begin{array}{lll}
1 & \sin \left(\omega k T+\varphi_{v}\right) & \cos \left(\omega k T+\varphi_{v}\right) \\
\sin (2 \omega k T)
\end{array}\right. \\
& \cos (2 \omega k T) \cdots \sin (N \omega k T) \cos (N \omega k T)]^{\mathrm{T}} \\
& \boldsymbol{W}=\left[\begin{array}{llllllll}
A_{0} & A_{1} & B_{1} & A_{2} & B_{2} & \cdots & A_{N} & B_{N}
\end{array}\right]^{\mathrm{T}}
\end{aligned}
$$

\section{SELECTIVE COMPENSATION}

The compensation method proposed in this paper is capable to selectively compensate harmonic currents generated by three-phase nonlinear unbalanced loads in fourwire systems. Because the compensation is achieved disregarding the displacement angle of each individual harmonic component, both active and reactive harmonic currents are compensated. The proposed method also compensates the fundamental reactive and the zero-sequence currents originated by the unbalanced load, thus providing sinusoidal balanced currents with null displacement angles at the point of common coupling of the active filter with the electric system.

The complete compensation scheme is presented in Fig. 3. This scheme is based on three adalines that determine the Fourier components of the three-phase load currents $i_{L a}, i_{L b}$ and $i_{L c}$. The errors $\varepsilon_{a}, \varepsilon_{b}$ and $\varepsilon_{c}$ are the differences between the Fourier approximated currents, which are the outputs of the neurons $\left(\hat{i}_{L a}, \hat{i}_{L b}\right.$ and $\left.\hat{i}_{L c}\right)$, and the measured load currents $\left(i_{L a}, i_{L b}\right.$ and $\left.i_{L c}\right)$.

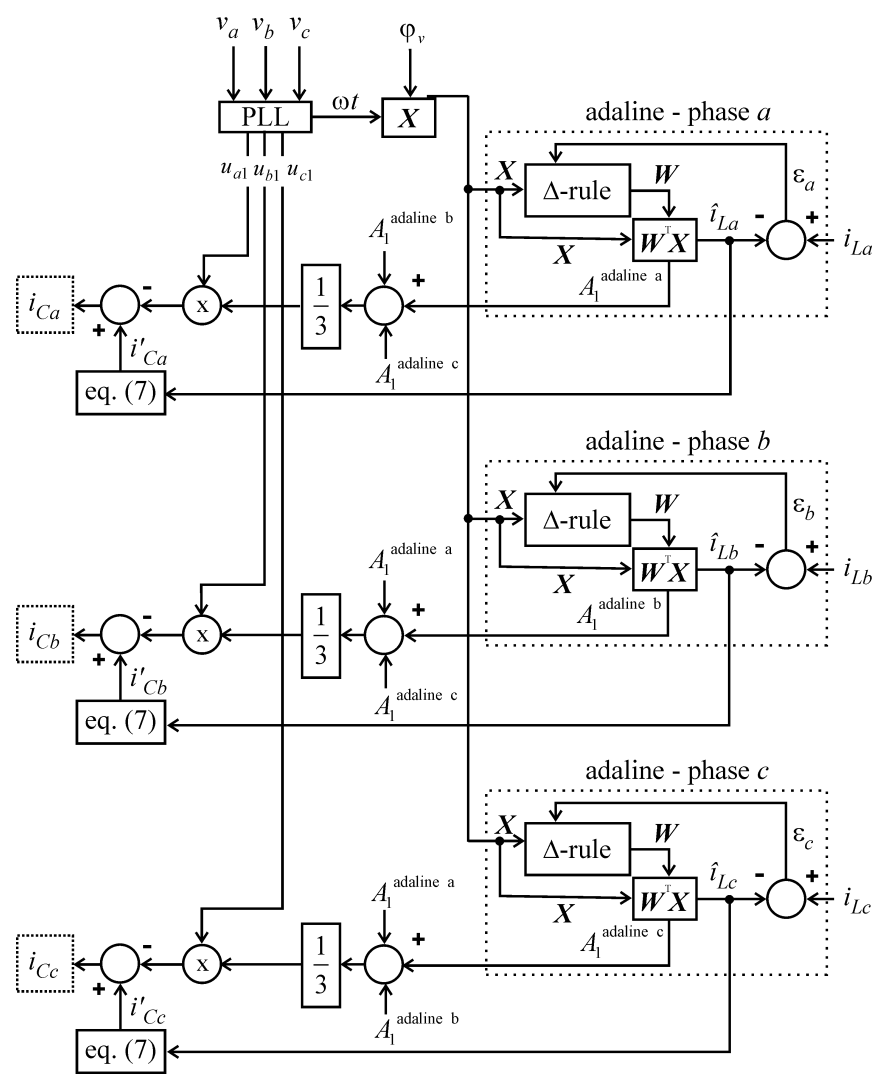

Fig. 3: Adaline-based four-wire current compensator.
Each adaline of the scheme of Fig. 3 provides a timevarying sum with the Fourier components of the estimated current. This sum is the product $\boldsymbol{W}^{\mathrm{T}} \boldsymbol{X}$ seen in (6).

$$
\begin{aligned}
\boldsymbol{W}^{\mathrm{T}} \boldsymbol{X}=A_{0} & +A_{1} \sin \left(\omega k T+\varphi_{v}\right)+B_{1} \cos \left(\omega k T+\varphi_{v}\right)+ \\
& +A_{2} \sin (2 \omega k T)+B_{2} \cos (2 \omega k T)+\cdots \\
& \cdots+A_{N} \sin (N \omega k T)+B_{N} \cos (N \omega k T)
\end{aligned}
$$

The angle $\varphi_{v}$ which appears in equations (4), (6) and (7) corresponds to the displacement of the phase voltage. This angle must be correctly adjusted according to the phase where the adaline is employed. When $\varphi_{v}$ is properly set the $2^{\text {nd }}$ and the $3^{\text {rd }}$ elements of $\boldsymbol{W}^{\mathrm{T}} \boldsymbol{X}$ are the two components of the fundamental phase current, both referenced to the angle of the respective phase voltage.

The selective harmonic compensation is achieved by selecting elements of the sum $\boldsymbol{W}^{\mathrm{T}} \boldsymbol{X}$ of each adaline. These elements are used in (7) to determine the three-phase preliminary compensation currents $\left(i_{C a}^{\prime}, i_{C b}^{\prime}\right.$ and $\left.i_{C c}^{\prime}\right)$. In (7), for instance, only the $3^{\text {rd }}, 5^{\text {th }}, 7^{\text {th }}, 9^{\text {th }}$ and $11^{\text {th }}$ harmonic components are selected.

$$
\begin{gathered}
i_{C\{a, b, c\}}^{\prime}=A_{0}+A_{1} \sin \left(\omega k T+\varphi_{v}\right)+B_{1} \cos \left(\omega k T+\varphi_{v}\right)+ \\
+\sum_{n=3,5,7,9,11} A_{n} \sin (n \omega k T)+\sum_{n=3,5,7,9,11} B_{n} \cos (n \omega k T) \\
i_{C\{a, b, c\}}=i_{C\{a, b, c\}}^{\prime}-i_{\{a, b, c\} 1}
\end{gathered}
$$

In equation (8) the fundamental components are subtracted from the preliminary compensation currents, yielding $i_{C a}, i_{C b}$ and $i_{C c}$, which are the compensation currents of the active power filter.

$$
i_{\{a, b, c\} 1}=u_{\{a, b, c\}_{1}}\left(\frac{A_{1}^{\text {adaline } \mathrm{a}}+A_{1}^{\text {adaline } \mathrm{b}}+A_{1}^{\text {adaline } \mathrm{c}}}{3}\right)
$$

The fundamental currents (that must not be compensated) are determined by equation (9), where fundamental unitary voltage signals are multiplied by the average of the fundamental active (non-displaced with reference to the phase voltage) load current amplitudes ( $A_{1}$ terms) determined by the adalines. Signals $u_{a 1}, u_{b 1}$ and $u_{c 1}$ have unitary amplitudes and are provided by a phase-locked loop (PLL) system, as shown later.

The compensation currents calculated by equation (8) are used to determine the positive- and negative-sequence compensation currents of the active filter expressed in $\alpha$ and $\beta$ variables, according to Fig. 4. If neutral currents are present in the system (originated by unbalanced loads) the compensation currents must have a zero-sequence component, which is directly obtained from the load currents (see Fig. 4) with equation (10). Currents $i_{C \alpha}, i_{C \beta}$ and $i_{C 0}$ shown in Fig. 4 are the final compensation currents of the active filter expressed in $\alpha \beta 0$ variables. The power invariant abc $\rightarrow \alpha \beta 0$ transformation used in this work is show in equation (A.1). 


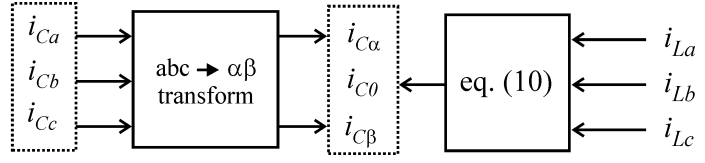

Fig. 4: Insertion of the zero-sequence component.

$$
i_{C 0}=i_{L 0}=1 / \sqrt{3}\left(i_{L a}+i_{L b}+i_{L c}\right)
$$

It is very convenient to have the compensation currents expressed in $\alpha \beta 0$ variables because the current controller (explained in a further section) used in this research is based on the stationary $\alpha \beta 0$ reference frame. Of course the compensation currents may be optionaly expressed in abc variables.

\section{FOUR-WIRE SHUNT ACTIVE FILTER}

\section{A. General scheme}

Fig. 5 shows the scheme of the shunt active power filter for three-phase four-wire systems studied in this research. It is basically composed of a current control system (whose main part is the current controller) and the selective current compensator based on adalines described in the previous section. The voltage controller is used to regulate the voltages $V_{C 1}$ and $V_{C 2}$ of the DC link capacitors, which must be kept constant and equalized.

\section{B. Current control system}

The current control system of the active power filter is composed of a three-leg voltage source inverter (VSI), a twocapacitor DC bus with neutral connection and a current controller (implemented with a DSP - digital signal processor). Fig. 6 shows the structure of the VSI. Fig. 7 shows the scheme of the current controller, which employs three proportional and integral (PI) regulators to control the compensation currents in the $\alpha \beta 0$ reference frame.

The control equations of the scheme of Fig. 7 are found in (11) and (12). One should notice that neither signals $\Delta i_{C \alpha}$, $\Delta i_{C \beta}$ and $\Delta i_{C O}$ (provided by the voltage controller) nor constants $k_{1}$ and $k_{2}$ are shown in Fig. 7. Constants $k_{1}$ and $k_{2}$ must be used so that the signals processed by the control equations have unitary amplitudes, which is necessary to correctly scale the outputs of the PI regulators, since the 3-D SVPWM modulator works with unitary inputs. Fig. 8 shows the simplified scheme of a 3-D space vector pulse-width modulation (SVPWM) modulator.

$$
\begin{gathered}
{\left[\begin{array}{l}
\varepsilon_{\alpha} \\
\varepsilon_{\beta} \\
\varepsilon_{0}
\end{array}\right]=k_{1}\left[\begin{array}{l}
i_{C \alpha}^{*} \\
i_{C \beta}^{*} \\
i_{C 0}^{*}
\end{array}\right]-\left[\begin{array}{l}
\Delta i_{C \alpha} \\
\Delta i_{C \beta} \\
\Delta i_{C 0}
\end{array}\right]-k_{2}\left[\begin{array}{l}
i_{C \alpha} \\
i_{C \beta} \\
i_{C 0}
\end{array}\right]} \\
{\left[\begin{array}{l}
u_{\alpha} \\
u_{\beta} \\
u_{0}
\end{array}\right]=\left(k_{p}+\frac{k_{i}}{s}\right)\left[\begin{array}{l}
\varepsilon_{\alpha} \\
\varepsilon_{\beta} \\
\varepsilon_{0}
\end{array}\right]}
\end{gathered}
$$

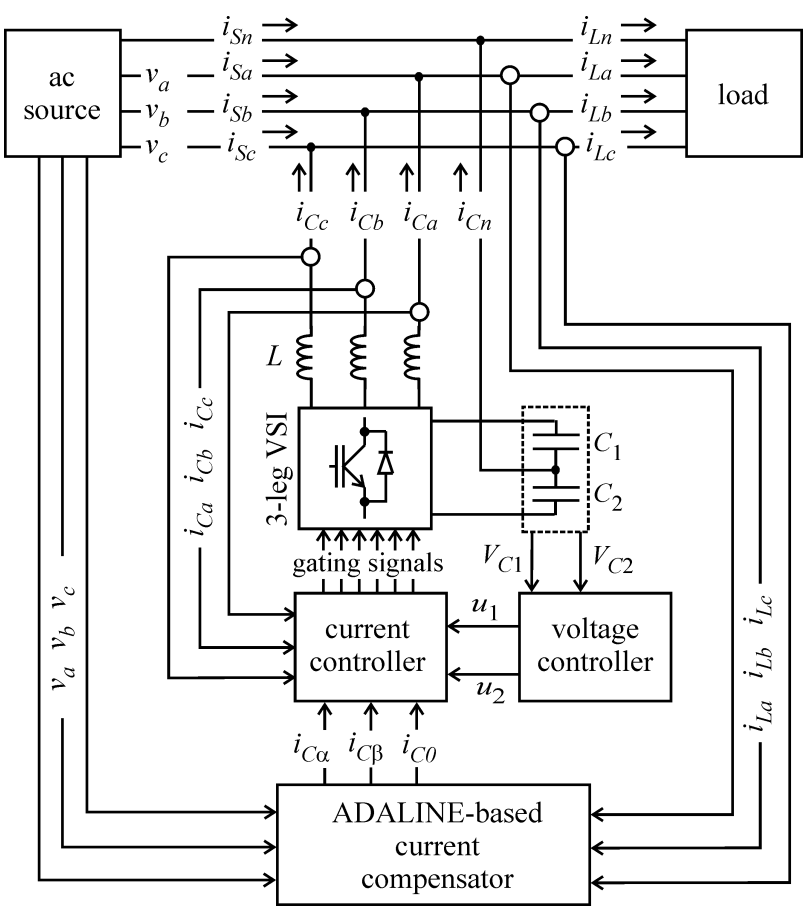

Fig. 5: Four-wire shunt active power filter.

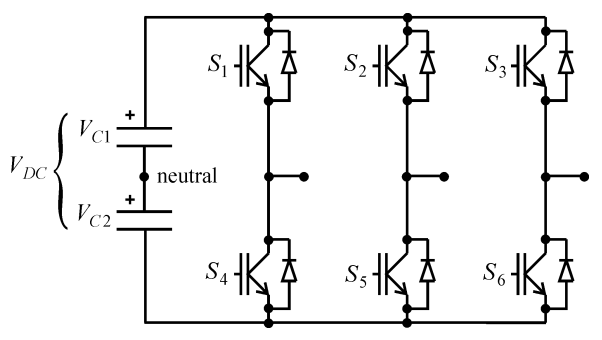

Fig. 6: Three-leg VSI with neutral connection.

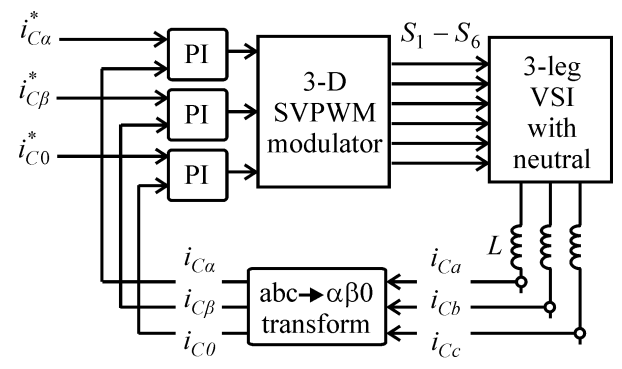

Fig. 7: Current controller with PI regulators.

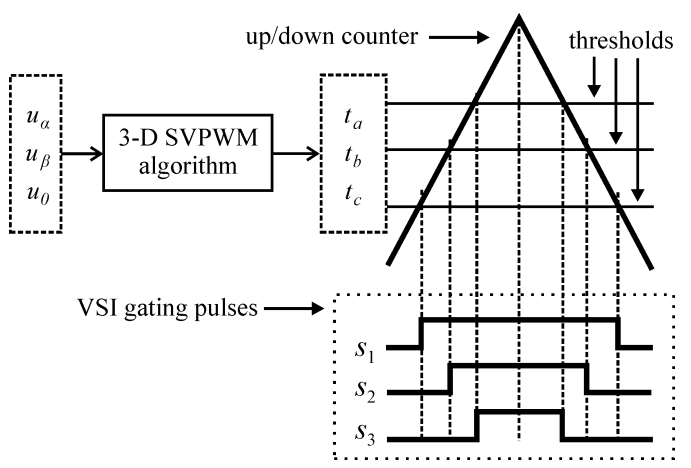

Fig. 8: Scheme of a 3-D SVPWM modulator. 
Equations (13) and (14) show a simple rule for the determination of the proportional and integral gains of (12), where $k_{p w m}$ is the gain of the VSI inverter with 3D-SVPWM, $\omega_{c}$ is the closed-loop crossover frequency, and $\phi_{m}$ is the margin phase. The parameters of Table 1 were used to adjust the PI regulators.

$$
\begin{gathered}
k_{p}=\left(\omega_{c} L\right) /\left(k_{p w m} k_{2}\right) \\
k_{i}=k_{p} \omega_{c} / \tan \phi_{m}
\end{gathered}
$$

\section{TABLE 1}

\begin{tabular}{l|l}
$L$ & $2 \mathrm{mH}$ \\
\hline$V_{D C}$ & $300 \mathrm{~V}$ \\
\hline$\omega_{c}$ & $9.4248 \mathrm{e}+003 \mathrm{rad} / \mathrm{s}$ \\
\hline$k_{2}$ & 0.1 \\
\hline$k_{p w m}{ }^{(*)}$ & 244.949 \\
\hline$\phi_{m}$ & $1.2217 \mathrm{rad}$ \\
& $=\left(V_{D C} / 2\right) /(\sqrt{6} / 4)$
\end{tabular}

The 3-D SVPWM modulator used in this current controller is based on a two-layer feedforward neural network. There would not be enough space in this paper to present a full discussion about 3-D SVPWM. References [10-11] present a detailed study about the design and the implementation of a four-wire current controller with 3DSVPWM. Fig. 9 shows a two-layer feedforward neural network (which is quite different of the adaline) that can entirely replace the modulation algorithm of Fig. 8.

The neural network of Fig. 9 is of the offline trained type (different of the adaline). Reference [10] describes the design and the training process of this neural network. After trained it can be easily implemented on a digital processor with equations (15) and (16).

$$
\begin{gathered}
\boldsymbol{Y}^{1}=\left[\begin{array}{ccc}
\frac{-2865}{15086} & \frac{2365}{3409} & \frac{802}{1067} \\
\frac{-309}{634} & \frac{-427}{697} & \frac{-581}{4180} \\
\frac{727}{908} & \frac{375}{9067} & \frac{285}{1888}
\end{array}\right]\left[\begin{array}{l}
u_{\alpha} \\
u_{\beta} \\
u_{0}
\end{array}\right]+\left[\begin{array}{c}
\frac{905}{5839} \\
\frac{-1037}{2714} \\
\frac{-167}{1730}
\end{array}\right] \\
{\left[\begin{array}{l}
t_{a} \\
t_{b} \\
t_{c}
\end{array}\right]=\left[\begin{array}{ccc}
\frac{-266}{911} & \frac{-497}{1289} & \frac{-1699}{2088} \\
\frac{-1201}{2947} & \frac{-589}{4478} & \frac{1063}{4459} \\
\frac{-939}{1936} & \frac{-1266}{1087} & \frac{-1009}{1773}
\end{array}\right] \boldsymbol{Y}^{1}+\left[\begin{array}{c}
\frac{351}{5062} \\
\frac{471}{1219} \\
\frac{-1630}{9323}
\end{array}\right]}
\end{gathered}
$$

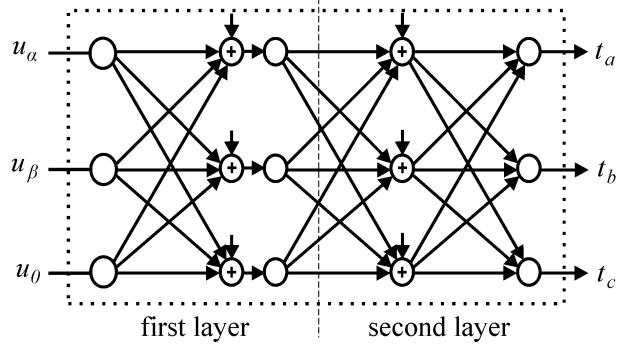

Fig. 9: Two-layer neural network used to replace the 3-D SVPWM algorithm.

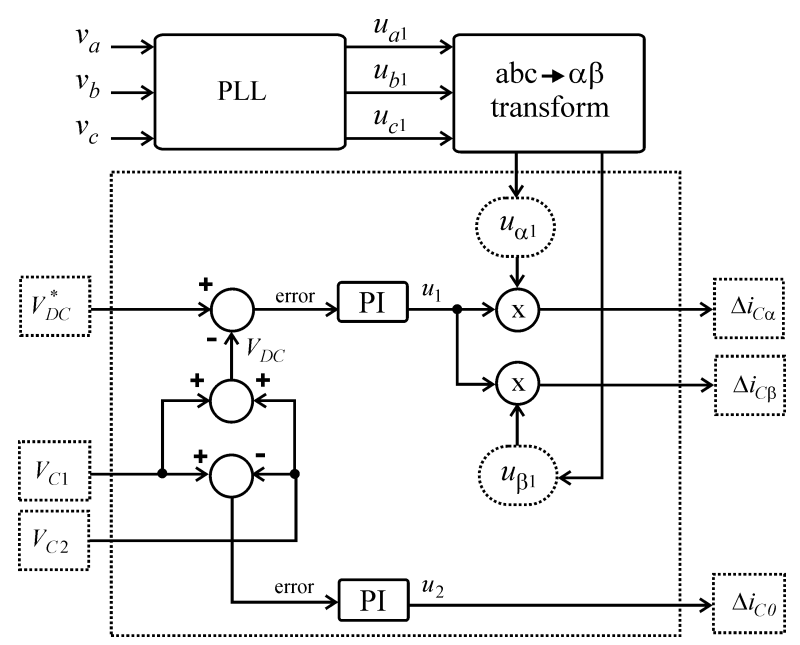

Fig. 10: Voltage controller.

\section{Voltage controller}

The signals $\Delta i_{C \alpha}, \Delta i_{C \beta}$ and $\Delta i_{C o}$ are subracted from the reference compensation currents of the current controller $\left(i_{C \alpha}, i_{C \beta}\right.$ and $\left.i_{C 0}\right)$. These signals are generated by the voltage controller for the maintenance of the voltage levels of the DC bus. Signals $\Delta i_{C \alpha}$ and $\Delta i_{C \beta}$ control the amplitudes of the positive- and negative-sequence components of the fundamental current of the active filter (and consequently of the source). The regulation of these amplitudes permits to control the voltage levels of the capacitors in order to keep them approximately constant. The regulation of the zerosequence current of the active filter with $\Delta i_{C O}$ permits to equalize the voltages of the capacitors $C_{1}$ and $C_{2}$. The voltage control equations are found in (17) and (18). Fig. 10 shows the scheme of the voltage controller.

$$
\begin{gathered}
{\left[\begin{array}{c}
\Delta i_{C \alpha} \\
\Delta i_{C \beta}
\end{array}\right]=\left(k_{p}+\frac{k_{i}}{s}\right)\left(V_{D C}^{*}-V_{D C}\right)\left[\begin{array}{l}
u_{\alpha 1} \\
u_{\beta 1}
\end{array}\right]} \\
\Delta i_{C 0}=\left(k_{p}+\frac{k_{i}}{s}\right)\left(V_{C 1}-V_{C 2}\right)
\end{gathered}
$$

\section{SYNCHRONIZATION WITH PLL}

A PLL appears in the schemes of the current compensator and of the voltage controller (figures 3 and 10). This PLL is used for the synchronization of the active power filter control 
(which includes current compensator, current controller and voltage controller) with the voltages of the electric source. In this work a one-phase PLL is used to generate three sinusoidal signals $\left(u_{a 1}, u_{b 1}, u_{c 1}\right)$ from the analysis of the phase-to-neutral voltage $v_{a}$. Fig. 11 shows the scheme of the PLL (implemented on a digital signal processor) which is a very simplified version of the PLL found in reference [12]. Indepth information about theory and operation of this PLL system may be found in [12].

Signals $u_{a 1}, u_{b 1}$ and $u_{c 1}$ have unitary amplitudes and are used in the adaline-based current compensator to extract the fundamental active components from the preliminary compensation currents. They are also used in the voltage controller to regulate the fundamental currents of the source and of the active filter. The angle $\theta=\omega t$ is used for the generation of the input vectors of the adalines (as seen in figures 2 and 4). This angle is synchronized with the angles of the source voltages, which makes the compensation of the active filter immune to frequency deviations since the adalines are capable to readjust their weight vectors whenever a new frequency condition is detected. When the frequency of the voltage source changes the input vector suffers modifications, which leads to a new (and brief) adapting process and consequently to a new set of weights. Furthermore, because the adalines are synchronized with the fundamental voltage signals $u_{a 1}, u_{b 1}$ and $u_{c 1}$ the compensation system is immune to voltage distortions. It is capable to provide sinusoidal and non-displaced currents at the electric source whatever are the waveforms of the phase voltages.

\section{SIMULATION RESULTS}

This section shows results of a shunt active power filter simulated with Matab/Simulink. Fig. 12 shows the load currents, Fig. 13 shows the compensation currents and Fig. 14 shows the compensated currents at the voltage source. Fig. 15 shows what happens when a load change occurs in phase $a$ : the adaline suffers a new adapting process and reaches a new steady state after a short period of time.

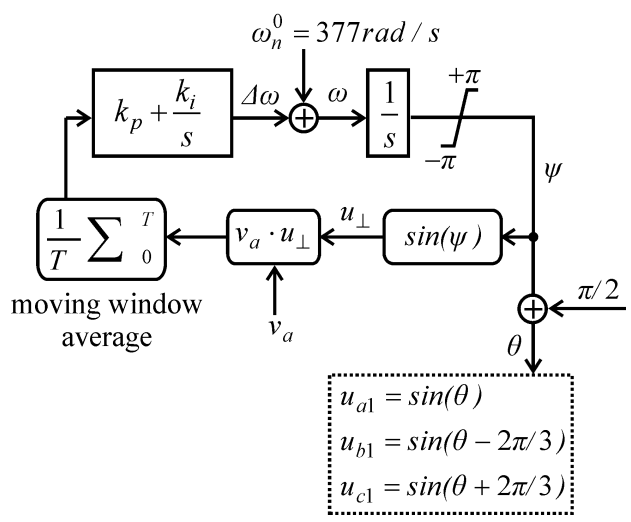

Fig. 11: Simplified one-phase PLL.

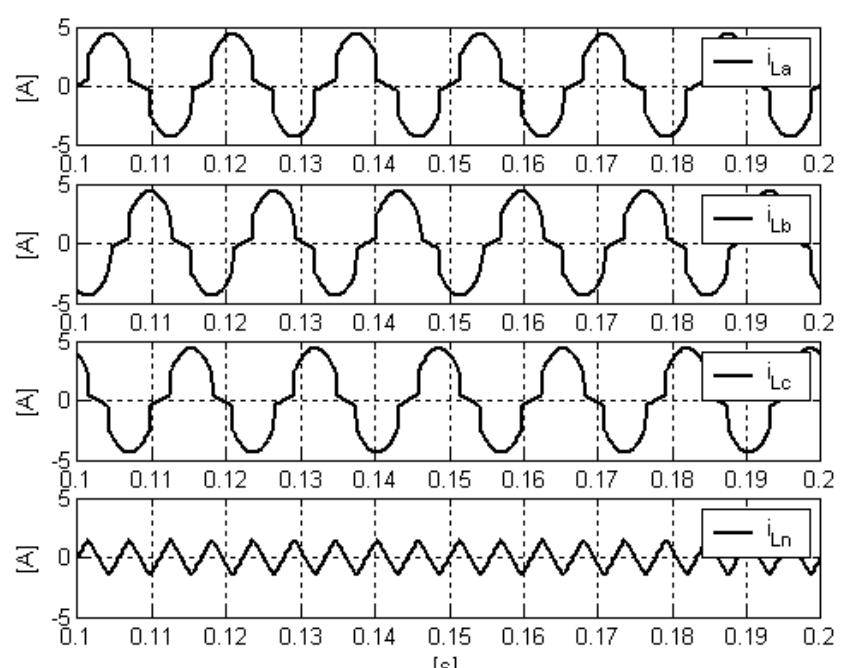

[s]

Fig. 12: Phase and neutral currents generated by a nonlinear load.
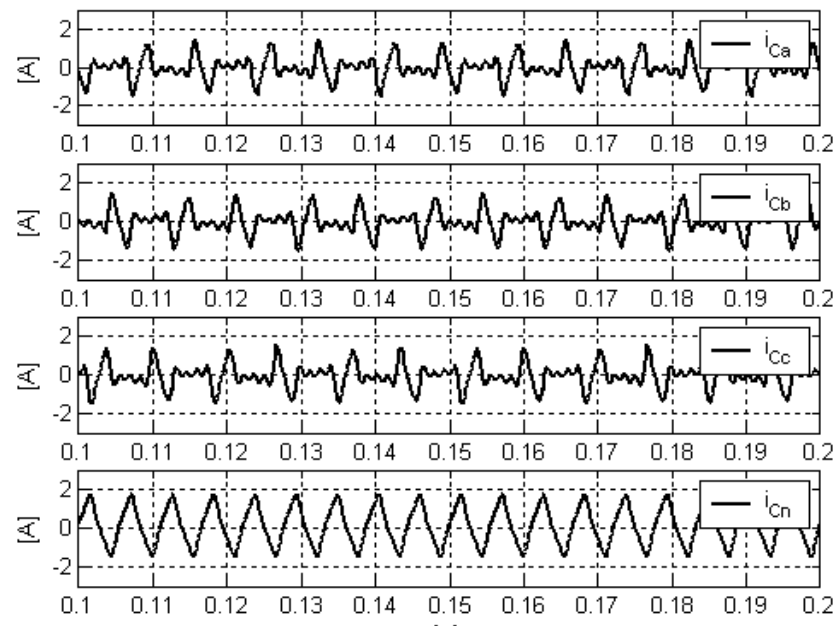

[s]

Fig. 13: Compensation currents of the active power filter.
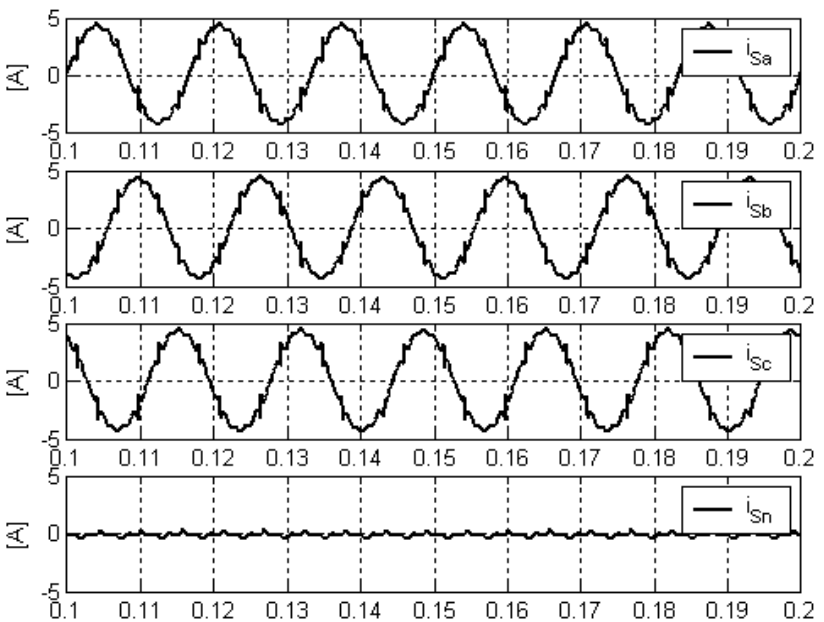

[s]

Fig. 14: Compensated currents at the source. 

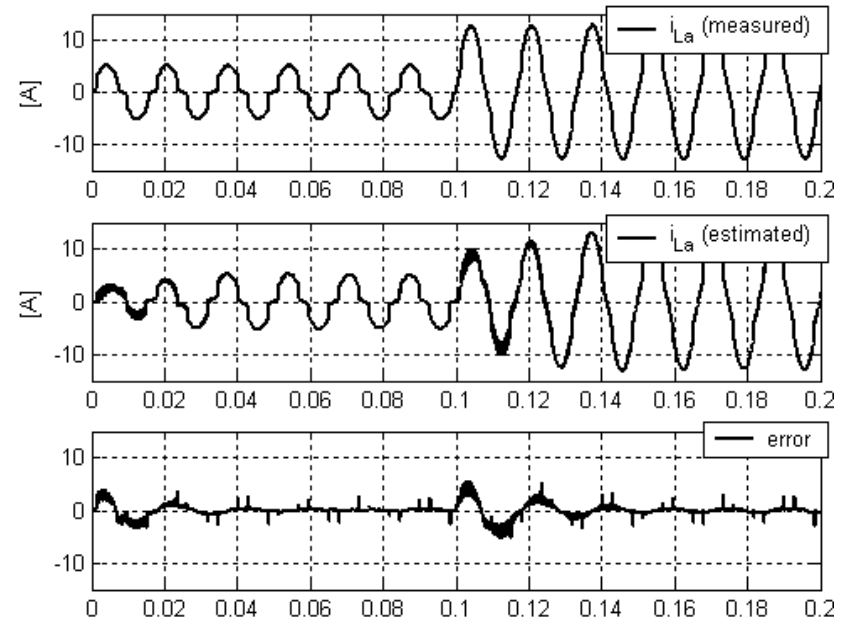

[s]

Fig. 15: Behavior of the adaline with load change: $i_{L a}, \hat{\imath}_{L a}, \varepsilon$.

TABLE 2

Total Harmonic Distortion (\%)

\begin{tabular}{l|c|c} 
& simulated & experimental \\
\hline Load current & 19.41 & 22.3 \\
\hline Compensated current & 8.24 & 10.45
\end{tabular}

\section{EXPERIMENTAL RESULTS}

Fig. 16 shows a picture of the experimental set-up of a four-wire shunt active power filter built in laboratory. The three-phase VSI is visible on the right top corner of the figure. At the center of the figure one sees the evaluation board with the TMX320F2812 fixed-point digital signal processor used in the implementation of the control algorithms. Fig. 17 shows the load current originated by a three-phase rectifier with diodes feeding a resistive load (top), the compensation current generated by the active filter (center) and the compensated current at the source (bottom).

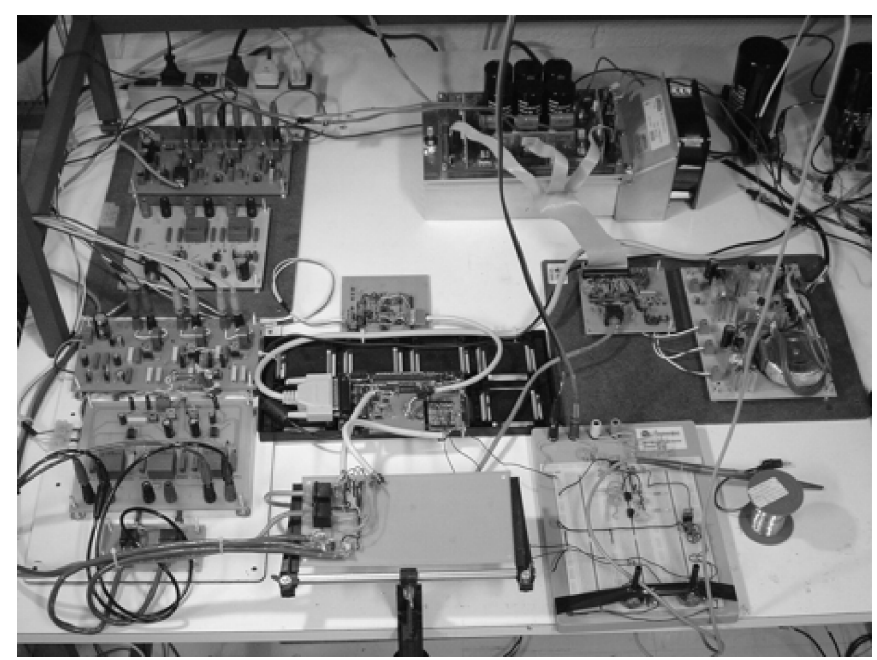

Fig. 16: Experimental setup of a shunt active power filter.

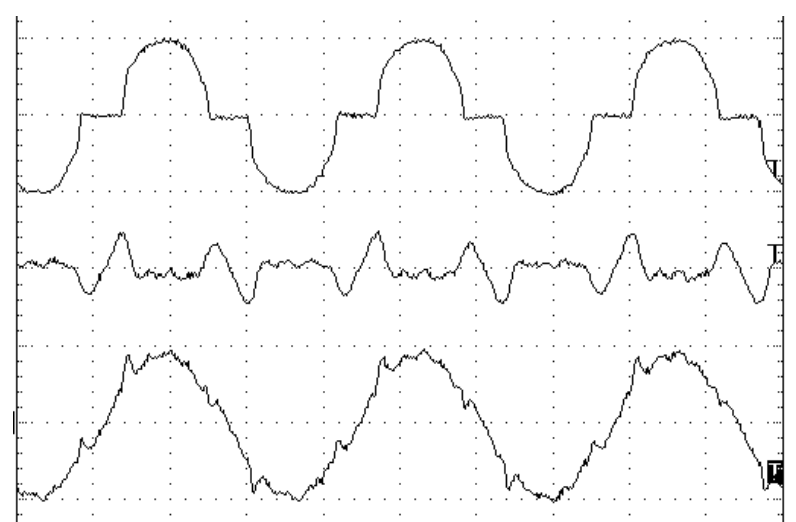

Fig. 17: Load current, compensation current and compensated current at the voltage source $(3 \mathrm{~A} / \mathrm{div}-5 \mathrm{~ms} / \mathrm{div})$.

The small peaks of the compensated currrent at the instants of load current transitions (i.e. when the rise and fall rates of the load currents are high) are primarily due to the fact that the compensation current does not (intentionally) contain higher harmonic components. Fig. 18 shows the neutral current originated by the load (top), the neutral current of the active power filter (center) and the compensated neutral current of the source (bottom). Fig. 19 shows the spectra of the load current (with several odd harmonic components), of the compensation current (with selected components only: $3^{\text {rd }}$ to $11^{\text {th }}$ ), and of the compensated current.

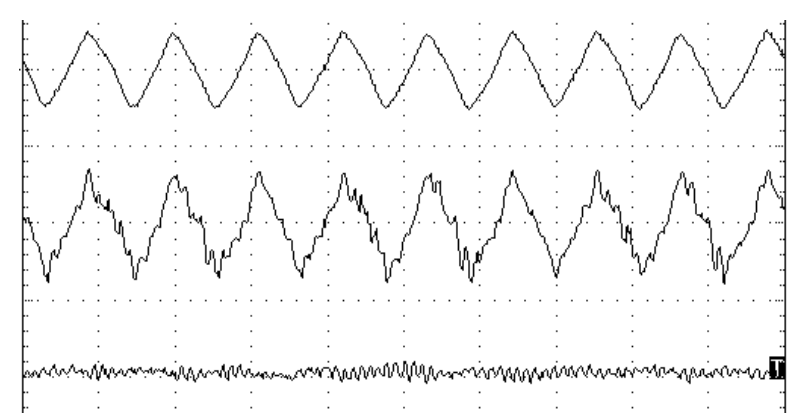

Fig. 18: Load neutral current, active filter neutral current and compensated source neutral current. $(3 \mathrm{~A} / \mathrm{div}-5 \mathrm{~ms} / \mathrm{div})$

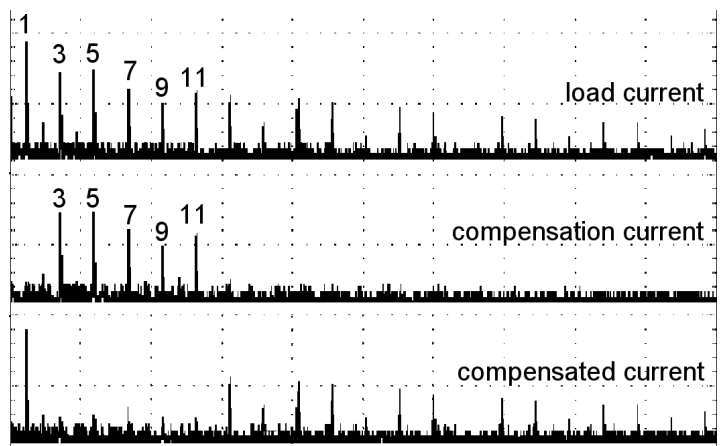

Fig. 19: Frequency spectra. $(40 \mathrm{~dB} / \mathrm{div}-250 \mathrm{~Hz} / \mathrm{div})$ 


\section{CONCLUSION}

This paper presents an alternative method for the control of a three-phase four-wire shunt active power filter employing neural networks. A simple and efficient four-wire selective current compensation strategy based on adaptive linear neural networks was studied and succesfully implemented on a fixed-point digital signal processor. In this compensation method harmonic components may be individually selected, avoiding excessive and unnecessary stress of the power converter. The theoretical complexity of this method is reasonably low, thus great programming effort is not required. Moreover its complexity and its computational burden are much smaller than those of the method proposed in [5], where many sine and cosine calculations and several other operations need to be performed. The compensation currents are injected into the electric system by a current controller whose pulse width modulation algorithm is based on a feedforward neural network, which allows easy implementation and fast execution of the current control algorithm. Altough other methods for the control of active filters are widely known, this work shows that neural networks are an interesting subject for research and for efficient application in power electronics.

\section{ACKNOWLEDGMENTS}

The authors are grateful to Dr. Fernando P. Marafão for his helpful and important comments, suggestions and assistance. The authors also thank Semikron (who kindly donated the $6 \mathrm{~kW}$ VSI used in the experimental set-up), and Texas Instruments (for the donation of the TMX320F2812 evaluation board). This work was supported by FAPESP (process number 02/05718-2).

\section{APPENDIX}

$$
\left[\begin{array}{c}
i_{\alpha} \\
i_{\beta} \\
i_{0}
\end{array}\right]=\sqrt{\frac{2}{3}}\left[\begin{array}{ccc}
1 & -1 / 2 & -1 / 2 \\
0 & \sqrt{3} / 2 & -\sqrt{3} / 2 \\
1 / \sqrt{2} & 1 / \sqrt{2} & 1 / \sqrt{2}
\end{array}\right]\left[\begin{array}{l}
i_{a} \\
i_{b} \\
i_{c}
\end{array}\right]
$$

\section{REFERENCES}

[1] R. El Shatshat, M. Kazerani, and M. M. A. Salama, "Modular approach to active power-line harmonic filtering", in Proceedings of the 29th Annual IEEE Power Electronics Specialists Conference (PESC), vol. 1, pp. 223-228, 1998.

[2] M. Rukonuzzaman and M. Nakaoka, "Adaptive neural network based harmonic current compensation in active power filter", in Proceedings of the International Joint Conference on Neural Networks (IJCNN), vol. 3, pp. 2281-2286, 2001.
[3] J. R. Vazquez and P. Salmeron, "Active power filter control using neural network technologies", in IEE Proceedings - Electric Power Applications, vol. 150, no. 2, pp. 139-145, 2003.

[4] F. P. Marafão, P. Mattavelli, S. Buso and S. M. Deckmann, "Repetitive-based control for selective active filters using discrete cosine transform", Revista da Sociedade Brasileira de Eletrônica de Potência, vol. 9, no. 1, pp. 29-36, 2004.

[5] P. Mattavelli and S. Fasolo, "A closed-loop selective harmonic compensation for active filters", in Proceedings of the 15th Annual IEEE Applied Power Electronics Conference and Exposition (APEC), vol. 1, pp. 399-405, 2000.

[6] S. Haykin, Neural Networks - A Comprehensive Foundation, Prentice Hall, 1998.

[7] M. T. Hagan, H. B. Demuth and M. H. Beale, Neural Network Design, PWS Publishing Company, 1995.

[8] B. Widrow and R. Winter, "Neural nets for adaptive filtering and adaptive pattern recognition", Computer, vol. 21, no. 3, pp. 25-39, 1988.

[9] B. Widrow and M. A. Lehr, "30 years of adaptive neural networks: perceptron, madaline, and backpropagation", in Proceedings of the IEEE, vol. 78, no. 9, pp. 14151442, 1990.

[10] M. G. Villalva, E. Ruppert F., "Current controller with artificial neural network for 3-phase 4-wire active filter", in Proceedings of the $35^{\text {th }}$ Annual IEEE Power Electronics Specialists Conference (PESC), vol. 2, pp. 993-998, 2004.

[11] M. G. Villalva, E. Ruppert F., "Current controller with 3-D SVPWM for 3-phase 4-wire active filters", IASME Transactions, vol. 1, no. 2, pp. 259-264, 2004.

[12] F. P. Marafão, S. M. Deckmann, J. A. Pomilio and R. Q. Machado, "A software-based PLL model: analysis and applications", in XV Congresso Brasileiro de Automática (CBA), 2004.

\section{ABOUT THE AUTHORS}

Marcelo Gradella Villalva graduated in electrical engineering at the University of Campinas (UNICAMP) in 2002. $\mathrm{He}$ received his master's degree and now pursues doctoral studies, both at UNICAMP.

Dr. Ernesto Ruppert Filho graduated in electrical engineering at the University of Campinas (UNICAMP) and received his master's and his doctorate in 1971, 1974, and 1983 respectively. Since 1972 he has been with UNICAMP as a faculty member and as a researcher. His widespread research interests include power electronics, superconductors, power systems, distributed generation, electric machines, and motor drives. 